\title{
Sex-Selective Effects on Behavior in a Mouse Model of Tuberous Sclerosis Complex
}

\author{
DRachel Michelle Saré, Abigail Lemons, Christopher Figueroa, Alex Song, Merlin Levine, and \\ DCarolyn Beebe Smith
}

https://doi.org/10.1523/ENEURO.0379-19.2020

Section on Neuroadaptation and Protein Metabolism, National Institute of Mental Health, National Institutes of Health, Department of Health and Human Services, Bethesda, MD 20814

\begin{abstract}
Tuberous sclerosis complex (TSC) is an autosomal dominant genetic disorder that is caused by a mutation in either TSC1 or TSC2. TSC affects multiple systems of the body, and patients with TSC display a range of neurologic and behavioral manifestations including seizures, intellectual disability, autism spectrum disorders, attention deficit hyperactivity disorder, anxiety, and mood disorders. Whereas behavioral phenotypes of many mouse models have been studied, the effects of sex have, for the most part, not been explored. We studied adult male and female Tsc2 heterozygous and control mice to investigate the influence of sex and genotype on behavior. On a test of social preference, Tsc2 heterozygous mice, regardless of sex, demonstrated lower preference for the stranger mouse than control mice. In the open field, Tsc2 heterozygous males and control females habituated to the open field with decreasing anxiety-like behavior over time, whereas Tsc2 heterozygous females did not show habituation to the open field environment. We did not find any statistically significant effects of genotype on open field activity, learning and memory or motor function. Our results highlight phenotype differences in Tsc2 heterozygous mice, some of which are influenced by sex. A consideration of how sex influences the behavioral phenotypes of TSC is critical to develop a more complete understanding of the disorder and better target future pharmacological treatments.
\end{abstract}

Key words: sex differences; tuberous sclerosis complex

\section{Significance Statement}

We investigated the role of sex on behavioral phenotypes in a mouse model of tuberous sclerosis complex (TSC). Our findings reveal potentially important sex differences in habituation to an anxiety-provoking environment. These results provide a more complete understanding of the disorder and highlight the need to investigate sex-specific differences to better target treatment of the disorder.

\section{Introduction}

Tuberous sclerosis complex (TSC) is an autosomal dominant neurogenetic disorder affecting approximately 1 in 6000 people. It is caused by a mutation in either TSC1

\footnotetext{
Received September 20, 2019; accepted March 31, 2020; First published April 10, 2020.

The authors declare no competing financial interests.

Author contributions: R.M.S. designed research; A.L., C.F., A.S., and M.L. performed research; R.M.S., A.L., and C.B.S. analyzed data; R.M.S., A.L., and C.B.S. wrote the paper.

This work was funded by the Intramural Research Program of the National Institute of Mental Health Grant ZIA MH00088936 DIRP. R.M.S. was also supported by a postdoctoral fellowship from Fragile X Research Foundation.
}

or TSC2 (European Chromosome 16 Tuberous Sclerosis Consortium, 1993; van Slegtenhorst et al., 1997). Multiple

Acknowledgements: We thank Zengyan Xia for genotyping the mice and Carrie Sheeler, Inna Loutaev, Isabella Maita, and Lee Harkless for performing preliminary experiments.

Correspondence should be addressed to Carolyn Beebe Smith at beebe@ mail.nih.gov.

https://doi.org/10.1523/ENEURO.0379-19.2020

Copyright (C) 2020 Saré et al.

This is an open-access article distributed under the terms of the Creative Commons Attribution 4.0 International license, which permits unrestricted use, distribution and reproduction in any medium provided that the original work is properly attributed. 
biological systems are affected in TSC, but the neurologic manifestations are often the most debilitating. TSC1 and TSC2 form a complex to inhibit the mammalian target of rapamycin (mTOR) pathway a regulatory node in cell growth and metabolism. The mTOR pathway is thought to play a key role in neuronal development and synaptic plasticity (Tavazoie et al., 2005; Jaworski and Sheng, 2006; de Vries and Howe, 2007), and consequently may be important in the unfolding of behavioral phenotypes.

Patients with TSC often experience seizures (80-90\%), learning difficulties (50\%), and autism spectrum disorders (50\%; Hunt and Shepherd, 1993; Thiele, 2004). Moreover, mutations in TSC2 tend to result in more severe neurologic phenotype (Au et al., 2007). Some studies of Tsc2 heterozygous mice have reported deficits on learning and memory tasks (Ehninger et al., 2008; Auerbach et al., 2011; Tang et al., 2014), although other studies have not (Potter et al., 2013; Reith et al., 2013). Tsc2 heterozygous mice have also displayed perseverative behavior (Potter et al., 2013) and social behavior abnormalities (Reith et al., 2013; Tang et al., 2014).

Sex may play a role in various phenotypes associated with TSC. Females are more likely to develop renal tumors than males (Hong et al., 2016). Lymphangioleiomyomatosis also occurs more commonly in female patients with TSC (Ryu et al., 2012). In addition, one epidemiological study indicated that male TSC patients were more prone to epilepsy and autism than female patients (Wataya-Kaneda et al., 2013); however, another study indicated that sex did not play a role in the central nervous system manifestations of TSC (Smalley, 1998). In most studies of mouse models of TSC, the effects of sex have not been investigated. Reported results are either from only male mice or from both sexes combined, but it is known that sex can have a profound role in both the morphology and function of the brain subsequently affecting behavioral output (Ngun et al., 2011).

In this study, we further investigated activity, anxietylike behavior, social preference, learning and memory, and motor coordination in groups of male and female control and Tsc2 heterozygous mice. Habituation to an anxiety-provoking environment (open-field) differed by genotype in a sex-dependent manner. We also found genotype differences in a test of social preference. We did not find genotype differences in open field activity, learning and memory, or motor function. Our results highlight important phenotypic differences, some of which are modulated by sex in Tsc2 heterozygous mice and illustrate that sex differences should be considered in future behavioral studies.

\section{Materials and Methods}

\section{Animals}

Tsc2 heterozygous mice (The Jackson Laboratory; B6; $129 S 4-T s c 2^{t m 1 D j k} / \mathrm{J}$ stock 004686) on a C57BL/6J background were maintained in house through mating of heterozygous and control breeder pairs. Animals were maintained in a climate-controlled central facility, on a standard 12/12 h light/dark cycle (lights on at 6 A.M.), with access to food and water ad libitum. was All procedures were approved by the National Institute of Mental Health animal care and use committee.

\section{Behavior testing}

Behavior testing began when the mice were between $75-85 \mathrm{~d}$ of age. All testing was done under normal lighting in the light phase. Testing was conducted in the following order: open field, novel object recognition (NOR), zero maze, social preference, and passive avoidance. Tests were spaced 3-4 d apart. We started with a cohort of 34 control males, 28 Tsc2 heterozygous males, 40 control females, and 29 Tsc2 heterozygous females. Every mouse underwent every test even if their data were not included. Data were not included because of prior exclusion criteria listed below, equipment malfunction, or testing performed outside of the time window presented. RotaRod and inkblot (spaced 2-3d apart) analyses were performed in a separate group of animals starting with 24 control males, 25 Tsc2 heterozygous males, 28 control females, and 30 Tsc2 heterozygous females. An additional experiment to examine a methodological detail in the social preference assay was conducted on an additional 48 control males. The timeline of testing is shown (Fig. 1).

\section{Open field}

To assess activity and anxiety-like behavior in response to a novel environment, mice were tested in an open field system (10.25-inch square clear Plexiglas chamber; Coulbourn Instruments) for $30 \mathrm{~min}$. We analyzed data in 6 -min epochs. Total horizontal distance traveled was used as a measure of activity and the ratio of distance traveled in the center ( $>6.25 \mathrm{~cm}$ from the wall) to total distance traveled was used as an inverse measure of anxiety-like behavior. Open field testing was performed between 9 A.M. and 1 P.M.

\section{NOR}

To assess learning and memory, mice underwent NOR testing. Objects were colored plastic interlocking toy bricks or plastic bottles of sand. Objects were similarly sized, but different in shape and color. Testing was conducted in the open field arena, so the open field testing was the habituation phase of NOR (Day 1). At the same time of day, on Day 2, the animal was placed in the open field arena with two identical objects (plastic interlocking bricks or bottles of sand) on opposite ends of the arena. The animal explored the objects for $5 \mathrm{~min}$. This training was repeated on Day 3. On Day 4, one of the objects was replaced by a novel object and the animal was allowed to explore for $5 \mathrm{~min}$. All sessions were video recorded, and assessment of time spent sniffing each object was analyzed. If the animal spent $<10$-s sniffing in total, it was excluded as the error in the measurements to determine discrimination would be high. A discrimination index was calculated as the difference between time spent sniffing the novel object and time spent sniffing the familiar object divided by the total time spent sniffing. 


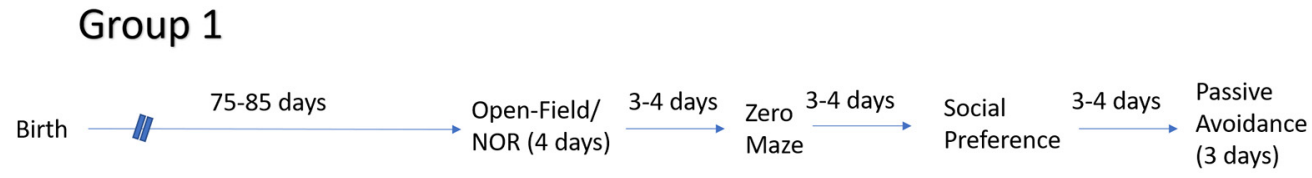

\section{Group 2}

Birth $\longrightarrow$ Rota-Rod $\stackrel{2-3 \text { days }}{\longrightarrow}$ Inkblot

Figure 1. Timeline of testing. We started with two cohorts of mice.

\section{Zero maze}

To assess anxiety-like behavior, the test mouse was placed on a zero maze (23.5-inch diameter ring with 2.75inch wide beams; Med Associates) facing the open section of the maze and allowed to explore for $5 \mathrm{~min}$. The amount of time spent in the open portion of the maze was determined. An animal was considered to be in a portion of the maze if both front paws were in that portion. If an animal fell off during the testing, it was eliminated from the analysis. Testing was performed between 12 and 5 P.M.

\section{Social preference}

Social preference was assessed with a three-chambered apparatus $(21.5 \times 16$-inch clear Plexiglas chamber equally divided into three compartments; Nadler et al., 2004). Testing was conducted in three parts (5 min each), with each phase immediately following the previous phase. (1) Habituation, the animal was habituated to the empty chamber. If an animal spent $>3$ min in one chamber, it was eliminated from the study for showing a side preference. We removed one control male, and three Tsc2 heterozygous females that demonstrated a side preference during habituation. (2) Sociability, an age/sex matched control stranger mouse was placed inside a social enclosure (inverted wire cup) in one chamber and an empty social enclosure was placed in the opposite chamber. The test mouse was placed in the middle chamber and allowed to freely explore. (3) Preference for social novelty, a novel age/sex matched stranger mouse was placed in the previously empty social enclosure and the test mouse once again was allowed to freely explore. Sniffing (the animal's nose directed toward the enclosure within $20 \mathrm{~mm}$ ) was determined from video recordings with the TopScan software (Clever Systems). Testing was initiated between 12 and 5:30 P.M.

\section{Passive avoidance}

Mice were tested on a passive avoidance system $(14 \times$ $7 \times 12$ inches; Coulbourn Instruments) with a 3-d protocol to assess learning and memory. Day 1, the animal was placed in the lighted chamber with the door to the dark chamber closed. After $30 \mathrm{~s}$ the door to the dark chamber was opened. Once the animal entered the dark chamber, the door closed automatically, and the test animal was returned to its home cage. Day 2, the animal was placed in the lighted chamber and after $30 \mathrm{~s}$, the door to the dark chamber was opened. Once the animal entered the dark chamber, the door closed, and the animal received a footshock $(0.3 \mathrm{~mA}, 1 \mathrm{~s})$. The animal was kept in the dark chamber for $15 \mathrm{~s}$ and then moved to a holding cage for $120 \mathrm{~s}$. The training session was then repeated, and the animal was returned to its home cage. Day 3, the animal was placed into the lighted chamber and after $30 \mathrm{~s}$, the door to the dark chamber was opened. The latency to enter the dark chamber (maximum of $570 \mathrm{~s}$ ) was recorded. Testing was performed at the same time of day across the testing sessions and occurred between 12 and 5 P.M.

\section{RotaRod}

To assess motor function, mice were placed on an accelerating RotaRod (Columbus Instruments). The acceleration was set at $0.1 \mathrm{rpm} / \mathrm{s}$. The time that the animal stayed on the RotaRod was measured and the average of two trials ( $1 \mathrm{~h}$ apart) was used for the analysis. Testing was initiated between 12 and 5 P.M.

\section{Inkblot}

To assess gait, non-toxic ink was placed on the fore(red) and hind-(black) paws of the mouse and the mouse was made to walk in a straight path along paper through a tunnel. The average length of the steps (measured from the heal of each hindpaw to the next) and the gait width was determined. Testing was performed between 1 and 5 P.M.

\section{Statistical analysis}

The data are reported as mean \pm SEM (standard error of the mean). Statistical analyses were performed using SPSS (IBM). NOR, zero maze, RotaRod, and passive avoidance data were analyzed by means of a two-way ANOVA with sex (male, female) and genotype (control, heterozygote) as between subjects' variables. Data from open field, social preference, and inkblot were analyzed by means of a mixed-model repeated measures ANOVA with sex (male, female) and genotype (control, heterozygote) as between subjects' variables and epoch (open field), chamber (social preference) and measure (inkblot) as within subjects' variables. ANOVA results are reported in Table 1. When appropriate, post hoc differences were assessed by means of Bonferroni-corrected $t$ tests. Effects with $p \leq 0.05$ are 
Table 1: ANOVA results

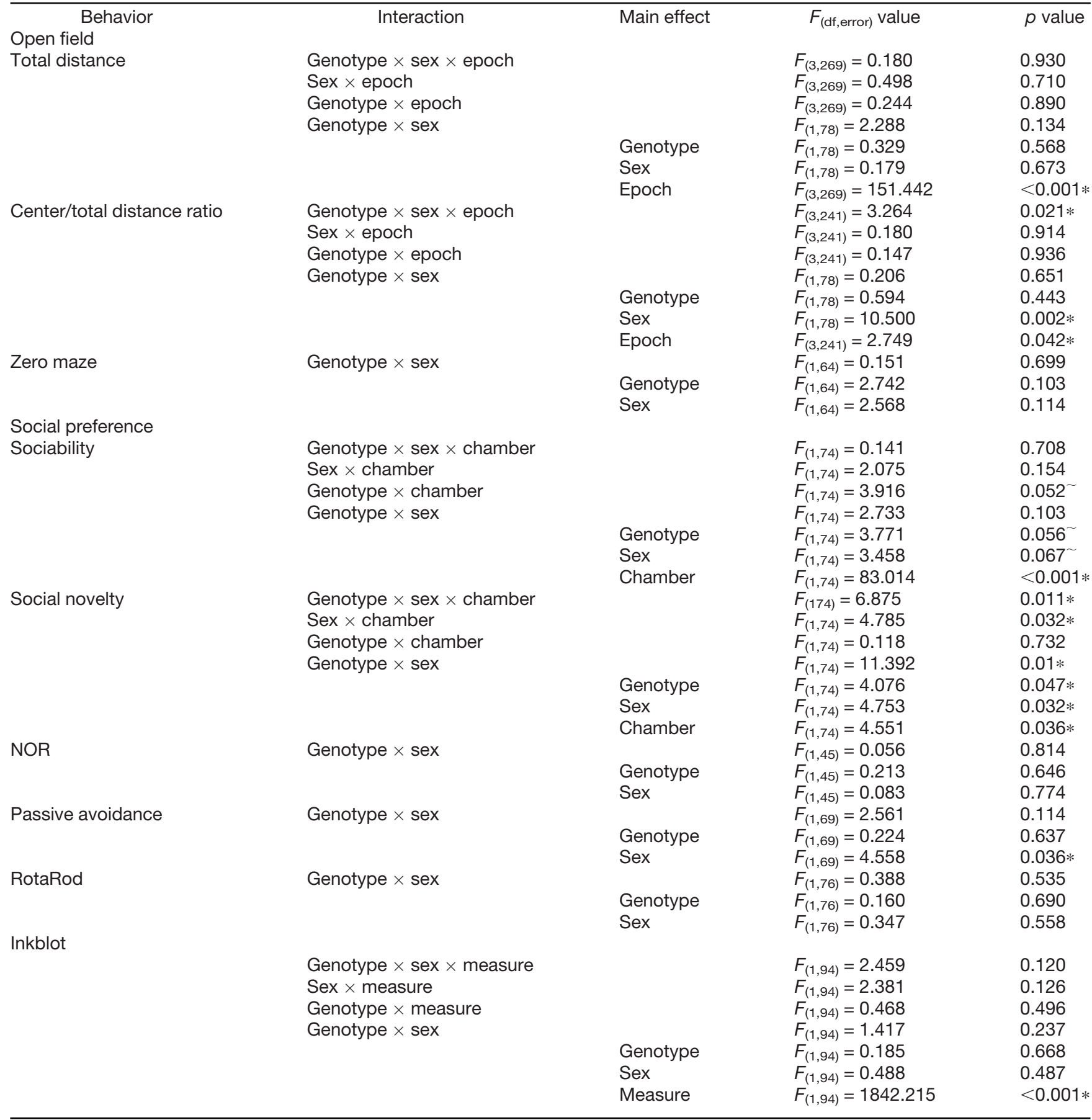

considered statistically significant and are reported with a $*$ symbol. Effects of $0.10 \geq p>0.05$ are also reported and denoted with $\mathrm{a} \sim$ symbol.

\section{Results}

\section{Open field activity}

We tested mice in a novel open field environment. None of the interactions were statistically significant (Table 1). The main effect of epoch was statistically significant as would be expected as animals habituated to the novel environment over time (Fig. 2).

\section{Anxiety-like behavior}

To assay anxiety-like behavior, we measured the ratio of distance traveled in the center to total distance traveled in the open field (Fig. $3 A$ ) and behavior in the zero maze (Fig. $3 B$ ). In the open field, the sex $\times$ genotype $\times$ epoch interaction was statistically significant $(p=0.021$; Table 1$)$. Post hoc tests show that, in control mice, male mice 


\section{Horizontal Distance}

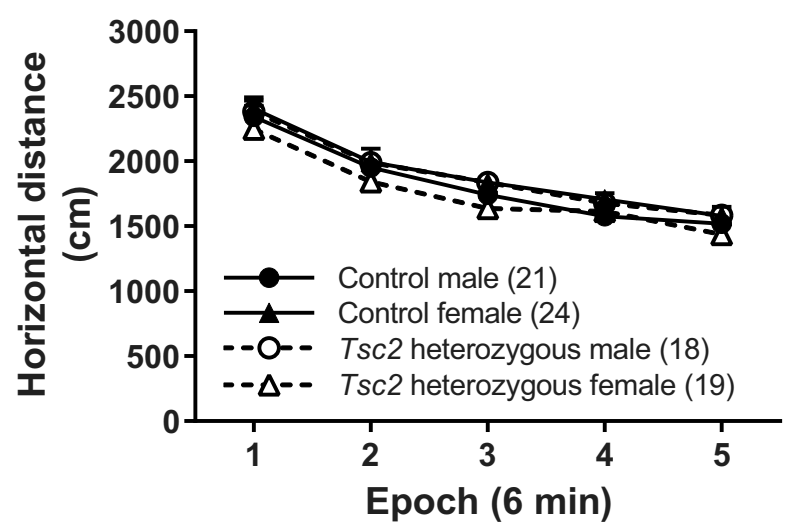

Figure 2. Activity levels. In distance traveled on the open field, we did not find any statistically significant main effects or interactions. Each point represents the mean \pm SEM for the number of animals indicated in parentheses.

traveled more relative distance in the center compared with female mice in epochs 1 and 2 ( $p=0.002$ for each epoch). In Tsc2 heterozygous mice, male mice traveled more relative distance in the center compared with female mice in epochs 2,3 , and $5(p=0.049, p=0.015$, and $p=0.008$, respectively; Fig. $3 A$ ). These data indicate that both Tsc2 heterozygous males and control females showed habituation to the environment over time with decreasing anxiety-like behavior. Tsc2 heterozygous females showed anxiety-like behavior at the beginning of the test and never habituated to the environment, whereas control males had less of an anxietylike response to the novel environment and did not show habituation. In the zero maze, we found no statistically significant interactions or main effects of sex or genotype (Table 1; Fig. 3B).

\section{Social preference}

We assayed social preference by means of a threechambered social preference task. In the sociability phase in which the test mouse could choose to interact with either an object (the empty container) or a stranger mouse (Fig. $4 A$ ), the genotype $\times$ chamber interaction approached statistical significance $(p=0.052$; Table 1$)$ suggesting that, regardless of sex, control mice demonstrated a greater preference for the mouse over the object than the Tsc2 heterozygous mice ( $p=0.016$; Fig. $4 A$ ).

In the preference for social novelty phase, the test mouse could interact with either the familiar mouse or a novel mouse (Fig. $4 B$ ); in this test, the genotype $\times$ sex $\times$ chamber interaction was statistically significant $(p=0.011$; Table 1). Post hoc analyses revealed that only female Tsc2 heterozygous mice spent more time sniffing the novel mouse than the familiar mouse $(p<0.001)$. Male Tsc2 heterozygous mice showed a statistically significant reduced sniffing time for the novel mouse compared with controls $(p=0.002)$, whereas female Tsc2 heterozygous mice showed a statistically significant increased time sniffing the novel mouse compared with controls $(p=0.018)$. Other statistically significant effects (not indicated on the figure; Fig. $4 B$ ) are that control male
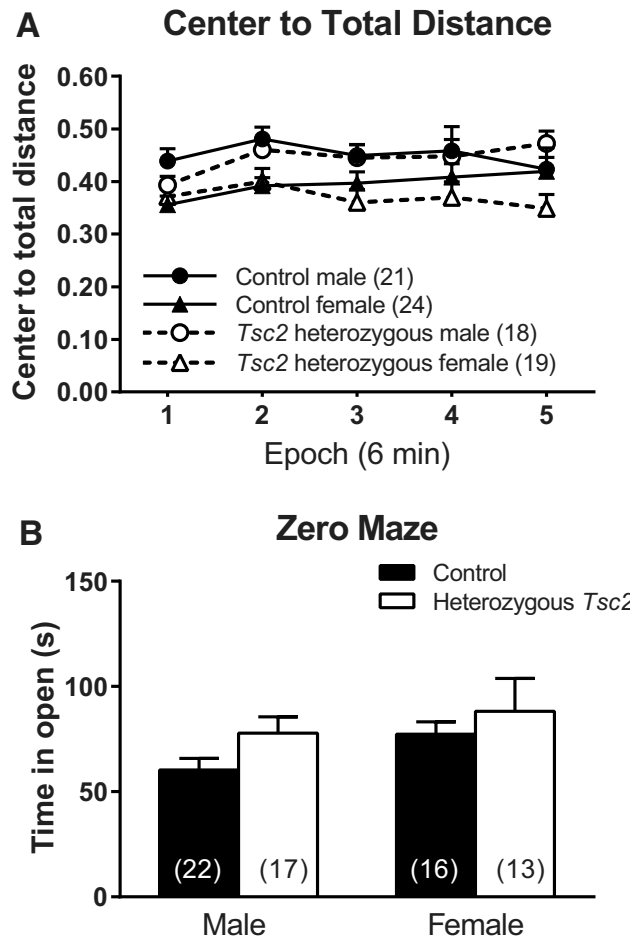

Figure 3. Anxiety-like behavior. $\boldsymbol{A}$, In the open field, we analyzed the ratio of distance traveled in the center of the open field to total distance traveled. The genotype $\times$ sex $\times$ epoch interaction was statistically significant. In control mice, males traveled more relative distance in the center than females in epochs 1 and 2 ( $p=0.002$ for each epoch). In Tsc2 heterozygous mice, males traveled more relative distance in the center than females in epochs 2 , 3 , and $5(p=0.049, p=0.015$, and $p=0.008$, respectively). Each point represents the mean \pm SEM for the number of mice indicated in parentheses. $\boldsymbol{B}$, We also analyzed anxiety-like behavior with the zero maze by measuring the time in the open portion of the maze. There were no statistically significant interactions or main effects. Bars represent mean \pm SEM for the number of animals indicated in parentheses.

mice spent more time sniffing both the familiar $(p=$ $0.013)$ and novel $(p=0.006)$ mice compared with control female mice, and Tsc2 heterozygous male mice spent less time sniffing the novel mouse than Tsc2 heterozygous females $(p=0.005)$.

\section{Learning and memory}

To assess learning and memory, we used NOR (Fig. 5A) and passive avoidance (Fig. $5 B$ ) tests. On the NOR, we did not find any statistically significant interactions or main effects (Table 1) likely due to the large variability in the measurements. On the passive avoidance task, we found a statistically significant $(p=0.036)$ main effect of sex, but no statistically significant effects with regard to genotype (Table 1) Overall, female mice, regardless of genotype, had a shorter latency than male mice (Fig. 5B).

\section{Motor function}

On the zero-maze task, we noticed that Tsc2 heterozygous mice tended to fall off more than control mice (11.4\% 
A

Sociability
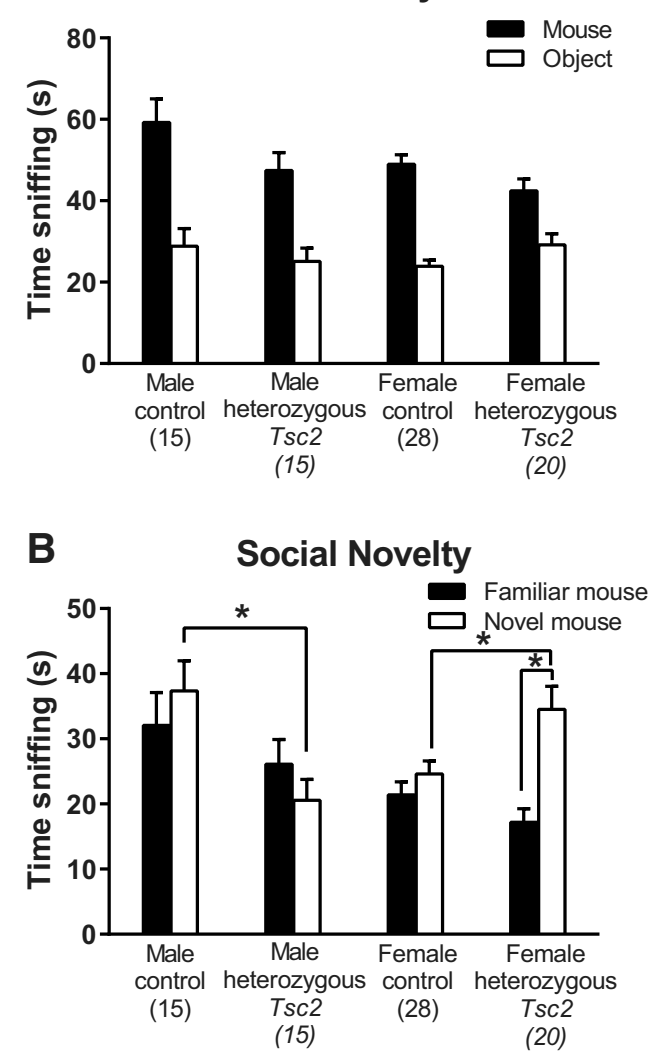

Figure 4. Social preference. $\boldsymbol{A}$, The chamber $\times$ genotype interaction approached statistical significance $(p=0.052)$. All mice, regardless of sex or genotype, sniffed the stranger mouse more than the object. Post hoc tests showed that control mice, regardless of sex, sniffed the social mouse more than Tsc2 heterozygous mice did $(p=0.016)$. Bars represent mean \pm SEM for the number of mice indicated in parentheses. $\boldsymbol{B}$, In the preference for social novelty test, the genotype $\times$ sex $\times$ chamber interaction was statistically significant $(p=0.011)$. Only Tsc2 heterozygous females sniffed the novel mouse statistically significantly more than the familiar mouse $(p<0.001$; paired post hoc tests). In males, Tsc2 heterozygous mice spent less time sniffing the novel mouse compared with control mice $(p=0.002)$. In females, Tsc2 heterozygous mice spent more time sniffing the novel mouse compared with control mice $(p=0.018)$. Other statistically significant effects (not indicated on the figure) are that control male mice spent more time sniffing both the familiar $(p=0.013)$ and novel $(p=0.006)$ mice compared with control female mice, and Tsc2 heterozygous male mice spent less time sniffing the novel mouse than Tsc2 heterozygous females $(p=0.005)$. Bars represent mean \pm SEM for the number of mice indicated in parentheses. $* p<0.05$.

of control males vs $21.4 \%$ of Tsc2 heterozygous males and $10 \%$ of control females vs $20.7 \%$ of Tsc2 heterozygous females). We sought to further investigate motor function/coordination with the RotaRod test (Fig. 6A) and the inkblot test (Fig. $6 B, C)$ to measure gait width and step length. On the RotaRod test none of the interactions or main effects were statistically significant (Table 1; Fig. 6A). On the inkblot test, there were no statistically significant differences regarding sex or genotype (Table 1; Fig. 6B,C).

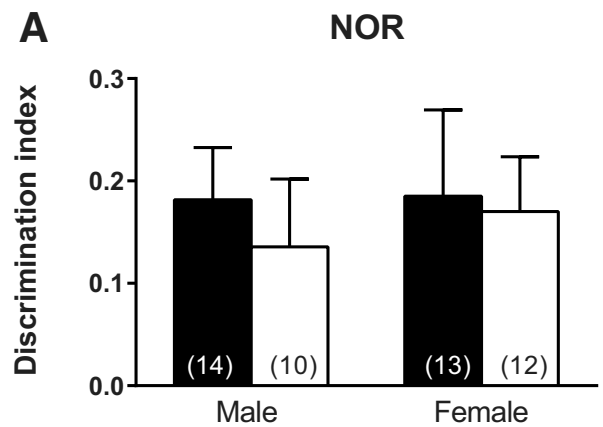

B Passive Avoidance

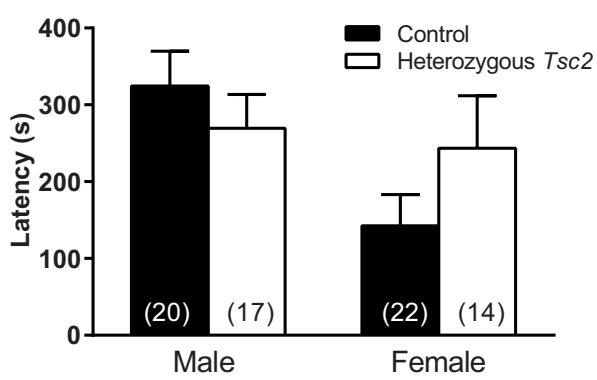

Figure 5. Learning and memory. $\boldsymbol{A}$, Results of NOR test do not show any statistically significant interaction or main effects. Bars represent mean \pm SEM of the number of mice indicated in parentheses. $\boldsymbol{B}$, In the passive avoidance test, the main effect of sex was statistically significant $(p=0.036)$, but effects of genotype were not. Overall, regardless of genotype, females had a lower latency to enter the dark side than males. Bars represent mean \pm SEM for the number of animals indicated in parentheses. The legend in $\boldsymbol{B}$ applies to both panels.

\section{Discussion}

The present study is, to our knowledge, the first study of the Tsc2 heterozygous mouse model of TSC to systematically examine sex differences in behavioral phenotype. In the open field, we saw differences in habituation to the novel environment with respect to anxiety-like behavior. Tsc2 heterozygous males and control females habituated to the open field with decreasing anxiety-like behavior over time, whereas Tsc2 heterozygous females did not show habituation to the open field environment. We also found genotype-specific differences in a test of social preference. We did not find any statistically significant effects of genotype on open field activity, learning and memory, or motor function. Our results highlight male/female differences in effects of a knock-out of a single allele of Tsc2 on some behaviors.

One of the limitations of our study is in the test of social behavior, specifically the response to social novelty. Neither male nor female control mice demonstrated a preference for the novel mouse. We have noted this behavior in prior studies in our lab. In the present study and in previous reports of a lack of social novelty preference, we note that the experimenter was not in the room during the test; mice were video recorded during the test, and results were analyzed later. In an ancillary experiment, we tested for an effect of the presence or absence of an experimenter present during the test. Results in control 
A

RotaRod

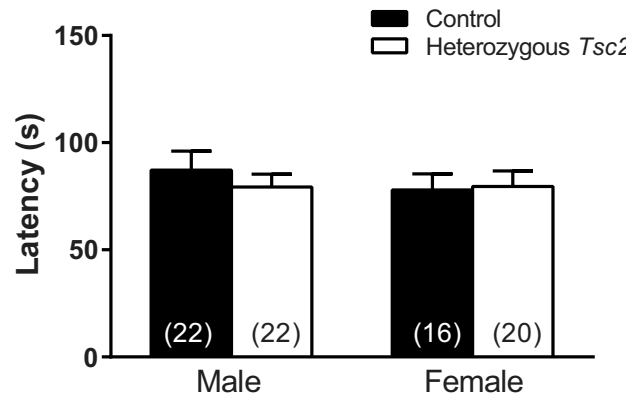

B Step Length

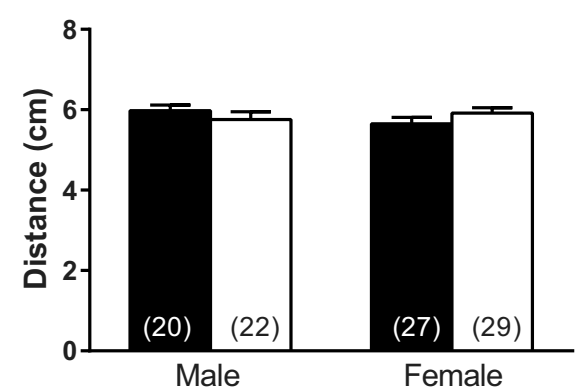

C

Step Width

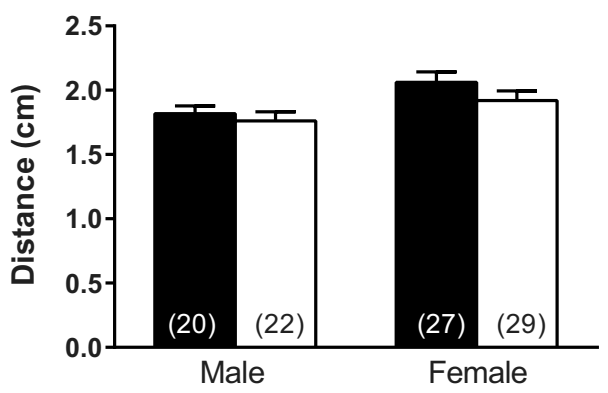

Figure 6. Motor function. A, Average latencies to fall off an accelerating RotaRod after two trials do not show any statistically significant interactions or main effects. Bars represent mean \pm SEM for the number of animals indicated in parentheses. $\boldsymbol{B}, \boldsymbol{C}$, Analysis of gait (step length and step width) show a statistically significant main effect of measure $(p<0.001)$ indicating that step width is less than step length. There were no statistically significant differences regarding sex or genotype. Bars represent mean \pm SEM for the number of animals indicated in parentheses. The legend in $\boldsymbol{A}$ applies to all three panels.

adult male C57BL/6J mice indicate that the preference for social novelty was demonstrated only when the experimenter was present (Fig. 7). It is interesting that in the original paper describing the three chambered task to measure preference for social novelty, the experimenter was present in the room (Nadler et al., 2004). This issue warrants further study.

Reports that did not account for sex did not find any anxiety-like behavior in Tsc2 heterozygous mice (Ehninger et al., 2008, 2012; Potter et al., 2013; Tang et al., 2014), though Tsc2 dominant negative mice (balanced mix of both sexes) did show a trend toward anxiety-like behavior as measured in the open-field (Ehninger and Silva, 2011). Our finding that
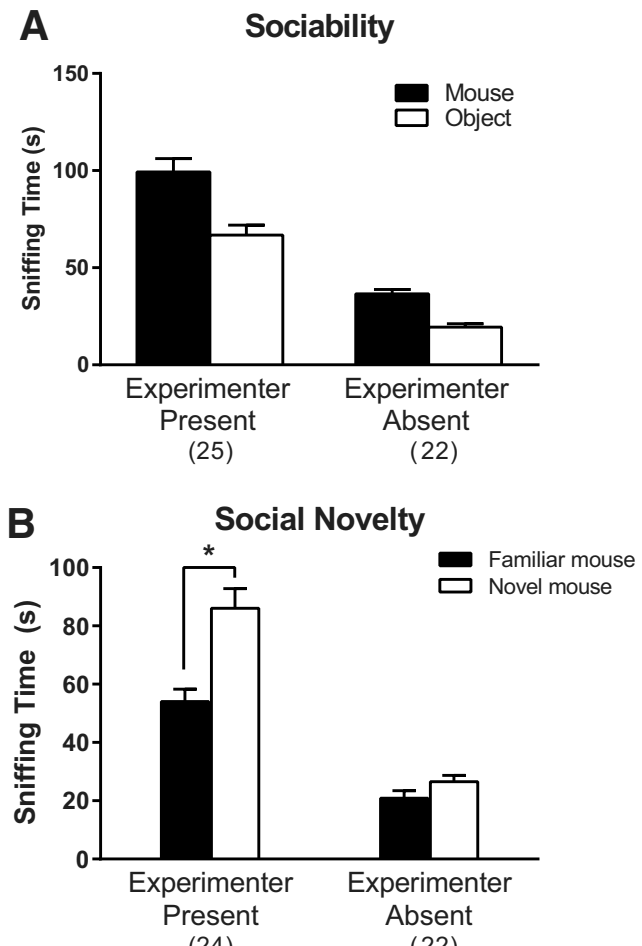

(24)

(22)

Figure 7. Social preference in control males in the presence or absence of the experimenter. We compared sociability and preference for social novelty whether or not the experimenter was present in the room (note: the male experimenter differed for each condition). $\boldsymbol{A}$, The experimenter presence $\times$ chamber interaction was not statistically significant, but both the main effect of experimenter presence $\left(F_{(1,45)}=243.1, p<0.0001\right)$ and chamber $\left(F_{(1,45)}=18.72, p<0.0001\right)$ were. $\boldsymbol{B}$, The experimenter presence $\times$ chamber interaction was statistically significant $\left(F_{(1,44)}=8.35, p=0.006\right)$. Post hoc $t$ tests showed that control males have a statistically significant preference for the novel mouse compared with the familiar mouse $(p<0.001)$ only if the experimenter is present. Bars represent mean \pm SEM for the number of mice indicated in parentheses. We tested 26 mice with the experimenter present and 22 mice with the experimenter absent. Data from one mouse during the sociability phase was lost. During the social novelty phase with the experimenter present, we lost one mouse because the door closed on his tail following habituation and another mouse because someone entered the room during the test. $* p<0.001$.

female, but not male, Tsc2 heterozygous mice did not habituate to the novel environment and maintained high anxiety levels throughout the test (did not adapt to the new environment) are of interest in view of the common occurrence of anxiety disorders in patients with TSC (25-60\%; Lewis et al., 2004; de Vries et al., 2007; Muzykewicz et al., 2007; Pulsifer et al., 2007). We did not observe genotype differences in anxiety per se in either the open-field or zero-maze tests in mice of either sex.

One specific point that we aimed to address in the present study was whether there were learning and memory deficits in Tsc2 heterozygous mice. It is generally believed that Tsc2 heterozygous mice show learning and memory deficits, but not every study has reported an abnormality. Some studies have shown that with the Morris water 
maze (MWM) test, Tsc1 or Tsc2 heterozygous animals have reduced number of crossings during the probe trial (Goorden et al., 2007; Ehninger et al., 2008), but others have shown no genotype difference in Tsc2 dominant negative mice on a C57BL/6 background (Chévere-Torres et al., 2012), Tsc2 heterozygous mice on a C57BL/6J 129 mixed background (Reith et al., 2013), and in a Tsc2 heterozygous rat model (Eker rats; Waltereit et al., 2011). In addition to MWM, learning and memory has been tested by means of the NOR test. Young Tsc2 heterozygous mice (C57BL/6J background) sniffed the novel object less than controls $30 \mathrm{~min}$ after exposure suggesting a deficit in short-term memory (Tang et al., 2014), but Tsc2 dominant negative mice (Chévere-Torres et al., 2012) nor Tsc2 heterozygous rats (Eker; Waltereit et al., 2011) showed a deficit on NOR with a $24 \mathrm{~h}$ delay before testing (long-term memory). We used two tests of learning and memory: NOR (recognition memory) and passive avoidance (fearbased learning and memory), and we saw no genotype differences on either test. We note that variability was very large on both tests as is often the case for behavior tests. Finally, although we observed a high proportion of Tsc2 heterozygous mice falling off the zero maze, we did not find any evidence of a deficit in motor performance. This is also consistent with previous reports. Baseline motor performance on a RotaRod test was not altered in Tsc2 dominant negative mice (Chévere-Torres et al., 2012). Additionally, Tsc1 and Tsc2 heterozygous mice did not show any differences in rotarod across the trial period (Sato et al., 2012; Tsai et al., 2012; Reith et al., 2013).

Here, we demonstrate sex-specific differences in habituation to an anxiety-provoking environment. These results suggest that female Tsc2 heterozygous mice do not habituate to a novel environment and maintain a high anxiety response. Our results highlight the importance of assessing males and females separately in behavioral testing. Our finding of some phenotypic differences in male and female Tsc2 heterozygous mice may yield insight into treatments of the behavioral phenotype of the disorder.

\section{References}

Au KS, Williams AT, Roach ES, Batchelor L, Sparagana SP, Delgado MR, Wheless JW, Baumgartner JE, Roa BB, Wilson CM, SmithKnuppel TK, Cheung MY, Whittemore VH, King TM, Northrup H (2007) Genotype/phenotype correlation in 325 individuals referred for a diagnosis of tuberous sclerosis complex in the United States. Genet Med 9:88-100.

Auerbach BD, Osterweil EK, Bear MF (2011) Mutations causing syndromic autism define an axis of synaptic pathophysiology. Nature 480:63-68.

Chévere-Torres I, Maki JM, Santini E, Klann E (2012) Impaired social interactions and motor learning skills in tuberous sclerosis complex model mice expressing a dominant/negative form of tuberin. Neurobiol Dis 45:156-164.

European Chromosome 16 Tuberous Sclerosis Consortium (1993) Identification and characterization of the tuberous sclerosis gene on chromosome 16. Cell 75:1305-1315.

de Vries PJ, Howe CJ (2007) The tuberous sclerosis complex proteins-a GRIPP on cognition and neurodevelopment. Trends Mol Med 13:319-326. de Vries PJ, Hunt A, Bolton PF (2007) The psychopathologies of children and adolescents with tuberous sclerosis complex (TSC): a postal survey of UK families. Eur Child Adolesc Psychiatry 16:1624.

Ehninger D, Silva AJ (2011) Increased levels of anxiety-related behaviors in a Tsc2 dominant negative transgenic mouse model of tuberous sclerosis. Behav Genet 41:357-363.

Ehninger D, Han S, Shilyansky C, Zhou Y, Li W, Kwiatkowski DJ, Ramesh V, Silva AJ (2008) Reversal of learning deficits in a Tsc2+/- mouse model of tuberous sclerosis. Nat Med 14:843848.

Ehninger D, Sano Y, de Vries PJ, Dies K, Franz D, Geschwind DH, Kaur M, Lee YS, Li W, Lowe JK, Nakagawa JA, Sahin M, Smith K, Whittemore V, Silva AJ (2012) Gestational immune activation and Tsc2 haploinsufficiency cooperate to disrupt fetal survival and may perturb social behavior in adult mice. Mol Psychiatry 17:6270.

Goorden SM, van Woerden GM, van der Weerd L, Cheadle JP, Elgersma Y (2007) Cognitive deficits in Tsc1 +/- mice in the absence of cerebral lesions and seizures. Ann Neurol 62:648655.

Hong CH, Tu HP, Lin JR, Lee CH (2016) An estimation of the incidence of tuberous sclerosis complex in a nationwide retrospective cohort study (1997-2010). Br J Dermatol 174:1282-1289.

Hunt A, Shepherd C (1993) A prevalence study of autism in tuberous sclerosis. J Autism Dev Disord 23:323-339.

Jaworski J, Sheng M (2006) The growing role of mTOR in neuronal development and plasticity. Mol Neurobiol 34:205-219.

Lewis JC, Thomas HV, Murphy KC, Sampson JR (2004) Genotype and psychological phenotype in tuberous sclerosis. J Med Genet 41:203-207.

Muzykewicz DA, Newberry P, Danforth N, Halpern EF, Thiele EA (2007) Psychiatric comorbid conditions in a clinic population of 241 patients with tuberous sclerosis complex. Epilepsy Behav 11:506-513.

Nadler JJ, Moy SS, Dold G, Trang D, Simmons N, Perez A, Young NB, Barbaro RP, Piven J, Magnuson TR, Crawley JN (2004) Automated apparatus for quantitation of social approach behaviors in mice. Genes Brain Behav 3:303-314.

Ngun TC, Ghahramani N, Sánchez FJ, Bocklandt S, Vilain E (2011) The genetics of sex differences in brain and behavior. Front Neuroendocrinol 32:227-246.

Potter WB, Basu T, O’Riordan KJ, Kirchner A, Rutecki P, Burger C, Roopra A (2013) Reduced juvenile long-term depression in tuberous sclerosis complex is mitigated in adults by compensatory recruitment of mGluR5 and Erk signaling. PLoS Biol 11: e1001627.

Pulsifer MB, Winterkorn EB, Thiele EA (2007) Psychological profile of adults with tuberous sclerosis complex. Epilepsy Behav 10:402406.

Reith RM, McKenna J, Wu H, Hashmi SS, Cho SH, Dash PK, Gambello MJ (2013) Loss of Tsc2 in Purkinje cells is associated with autistic-like behavior in a mouse model of tuberous sclerosis complex. Neurobiol Dis 51:93-103.

Ryu JH, Sykes AM, Lee AS, Burger CD (2012) Cystic lung disease is not uncommon in men with tuberous sclerosis complex. Respir Med 106:1586-1590.

Sato A, Kasai S, Kobayashi T, Takamatsu Y, Hino O, Ikeda K, Mizuguchi M (2012) Rapamycin reverses impaired social interaction in mouse models of tuberous sclerosis complex. Nat Commun 3:1292.

Smalley SL (1998) Autism and tuberous sclerosis. J Autism Dev Disord 28:407-414.

Tang G, Gudsnuk K, Kuo SH, Cotrina ML, Rosoklija G, Sosunov A, Sonders MS, Kanter E, Castagna C, Yamamoto A, Yue Z, Arancio O, Peterson BS, Champagne F, Dwork AJ, Goldman J, Sulzer D (2014) Loss of mTOR-dependent macroautophagy causes autistic-like synaptic pruning deficits. Neuron 83:1131-1143. 
Tavazoie SF, Alvarez VA, Ridenour DA, Kwiatkowski DJ, Sabatini BL (2005) Regulation of neuronal morphology and function by the tumor suppressors Tsc1 and Tsc2. Nat Neurosci 8:1727-1734.

Thiele EA (2004) Managing epilepsy in tuberous sclerosis complex. J Child Neurol 19:680-686.

Tsai PT, Hull C, Chu Y, Greene-Colozzi E, Sadowski AR, Leech JM, Steinberg J, Crawley JN, Regehr WG, Sahin M (2012) Autistic-like behaviour and cerebellar dysfunction in Purkinje cell Tsc1 mutant mice. Nature 488:647-651.

van Slegtenhorst M, de Hoogt R, Hermans C, Nellist M, Janssen B, Verhoef S, Lindhout D, van den Ouweland A, Halley D, Young J,
Burley M, Jeremiah S, Woodward K, Nahmias J, Fox M, Ekong R, Osborne J, Wolfe J, Povey S, Snell RG, et al. (1997) Identification of the tuberous sclerosis gene TSC1 on chromosome 9q34. Science 277:805-808.

Waltereit R, Japs B, Schneider M, de Vries PJ, Bartsch D (2011) Epilepsy and Tsc2 haploinsufficiency lead to autistic-like social deficit behaviors in rats. Behav Genet 41:364-372.

Wataya-Kaneda M, Tanaka M, Hamasaki T, Katayama I (2013) Trends in the prevalence of tuberous sclerosis complex manifestations: an epidemiological study of 166 Japanese patients. PLoS One 8:e63910. 